\title{
OFDM Link Performance with Companding for PAPR Reduction in the Presence of Non-Linear Amplification
}

\author{
Thomas G. Pratt, Nathan Jones, Leslie Smee, and Michael Torrey \\ Georgia Tech Research Institute \\ Georgia Institute of Technology \\ Atlanta, Georgia, 30332 \\ Thomas.pratt@gtri.gatech.edu
}

\begin{abstract}
Use of companding for peak-to-average-power ratio (PAPR) control is explored for a link involving a non-linear transmit power amplifier with orthogonal frequency division multiplexing (OFDM). Specifically, the objective of the study was to determine if companding using $u$-law compression/expansion at the transmitter/receiver, respectively, provides end-to-end performance gains relative to a system without companding. We consider the use of companding to ameliorate the impact of nonlinearities in the transmit amplifier. In the absence of companding, transmitter operation near saturation raises the signal level at the receiver but, because of the nonlinearities in the amplifier response, also results in distortion that impacts overall link performance. As the transmit power is backed-off from saturation, amplifier distortion is reduced, but error components due to lower SNR at the receiver become more significant. When companding is introduced in the system, the system is able to operate closer to saturation without substantial transmit distortion. However, requisite expansion of the compressed signal at the receiver yields noise amplification which can counteract any of the performance gains that would otherwise accrue from the increased SNR at the receiver. At issue is whether or not operating conditions exist (e.g., backoff, SNR, amplifier linearity model, etc) for which companding enhances the end-to-end performance relative to the link performance without companding. System simulation models were employed using Rapp's nonlinear power amplification models, where average symbol distance errors were used as performance metrics. We found that companding can provide very modest performance gains in comparison to systems that do not employ companding. Performance trends were corroborated in a hardware testbed with an amplifier chain, where average bit error rates were experimentally determined.
\end{abstract}

Keywords: Amplifier Distortion, Companding, Communication System Performance

\section{Introduction}

Orthogonal Frequency Division Multiplexing (OFDM) is a firmly established air interface for fixed wireless, WLAN, and mobile wireless applications. Because OFDM is a multicarrier waveform, it may exhibit large peak-to-average power ratios (PAPR), which can result in saturation of the amplifier or alternatively encourage use of large back-offs that impact power efficiency and subsequently SNR at the receiver. Hence, methods to reduce PAPR are of interest, since they potentially can yield performance gains in these systems. Various PAPR reduction techniques have been proposed [4-9, 14-21], including techniques based on companding [4-9, 20, 21]. This paper investigates

This material is based upon work supported by The National Science Foundation under Grant No. 0121565. 
$u$-law companding and its impact on link performance in the presence of both high PAPR signals, nonlinear power amplification, amplifier backoff, and noise enhancement.

To improve link performance with any given amplifier, one potential strategy is to increase the transmit power, shifting the operating point towards amplifier saturation. However, while this increases the signal power at the receive end of the link, signals undergo distortion in the nonlinear operating region of the amplifier [1], and this impairment is aggravated for high PAPR-signals. The nonlinear amplifier distortion translates to spectral regrowth and to a subsequent increase in BER, potentially offsetting any performance gains derived from the increased received signal power level. The transmit power can be decreased by backing-off the operating point from saturation to reduce the amplifier distortion, but this is done at the expense of reducing the achieved signal-to-noise power ratio (SNR) at the receiver. Armada and Garcia [3] have suggested a practical way of specifying the optimal operating point for OFDM in the presence of nonlinear amplifiers. Their heuristic approach involves setting the backoff such that the spectral regrowth shoulders are $20 \mathrm{db}$ below the in-band signal level. In [2], backoffs of $8 \mathrm{db}$ are considered for the IEEE802.16.3c standard.

There is a design tradeoff in defining a useful operating point, and this will depend upon the characteristics of the power amplifier. PAPR reduction techniques may permit the transmitter to operate closer to saturation without substantially increasing signal distortion caused by the non-linear amplifier. In this work, we explore a simple PAPR reduction approach based on $u$-law companding. Companding is known to reduce the PAPR ratio of OFDM signals [4-9]. Hence, it would potentially allow transmission of the companded signal at a reduced backoff level (i.e., closer to saturation) for a comparable level of amplifier distortion, thus resulting in higher signal power and improved performance at the receiver.

There are some potential drawbacks to adopting signal compression techniques. One drawback is that signal compression results in spectral regrowth, a form of signal distortion similar to that which occurs with signal limiting (e.g. such as with hardlimiters). Filtering could be applied following compression to contain the spectral regrowth, but at the expense of increasing the PAPR [10]. Another undesired effect of $u$ law companding techniques, perhaps of greater concern, involves the requisite expansion of the compressed signal at the receiver, a process which amplifies receiver noise [4-8].

One of the first papers to deal with companding for PAPR reduction of OFDM signals was the work of Wang and his colleagues $[4,5,6]$. In their analysis, $u$-law companding was employed to reduce PAPR from OFDM signals to minimize A/D and D/A distortion, and to reduce symbol error rates. $u$-law companding proved to be beneficial in high SNR cases (e.g., SNR>35 db). When amplifier distortion is considered in an end-to-end systems model, we find that companding offers modest performance improvements with appropriate selection of $u$ and the amplifier backoff level, even at lower SNR.

In [7], Wang et al also investigated OFDM systems with A-Law companding and determined optimal A-law companding coefficients for different OFDM DFT sizes. In later work, Huang et al [8,9] investigated companding transforms , which combined advantages of both clipping and companding. Compared with conventional companding techniques, the transform was found to reduce PAPR more effectively. More recent investigations of companding have included studies exploring exponential companding [21] and a novel companding function by Jiang [19]. 
We consider the end-to-end performance of an OFDM link with and without companding and conduct analysis tradeoffs for system parameters including $u$, power amplifier nonlinear distortion, power amplifier backoff, and received signal-to-noiseratio. Such interdependencies between PAPR reduction techniques, power amplification characteristics, and noise amplification help to motivate this study, since an understanding of these provide a springboard for legitimizing the consideration of companding as a viable PAPR reduction technique for improving link performance in the presence of amplifier distortion.

\section{System Description}

The system that is analyzed in this work involves an OFDM air interface with system functions and impairments as illustrated in Figure 1. The transmitter section maps a random data bit sequence, $\mathbf{b}_{\mathrm{tx}}$, into a sequence of QAM symbols, $\mathbf{S}_{\mathrm{tx}}$. The QAM symbols are partitioned into N-length blocks and modulated onto the subcarriers of an OFDM modulator via the inverse Discrete Fourier Transform (IDFT). After guard interval insertion, the resulting OFDM signal is integrated into a frame that includes a preamble and training sequences for synchronization and channel estimation, respectively. Prior to transmission, the signal is companded with a $u$-law compander, and then scaled for power normalization. The scaled, companded signal is then passed through the transmit amplifier, which distorts the signal according to non-linear solid state power amplifier models [9].

Following transmission, the signal is corrupted with additive white Gaussian noise. Processing within the receiver includes synchronization, channel estimation and compensation, $u$-law expansion, and OFDM demodulation. The resulting QAM symbols are thencompared with the transmitted QAM symbols to form an error metric for performance comparisons. Error metrics used in this study include the average symbol error distance, which is used in the simulations to identify performance trends, and bit error rates in hardware testing to corroborate the observed trends.

The salient features of the signal and impairment models associated with this system are described in greater detail in the remainder of this section.

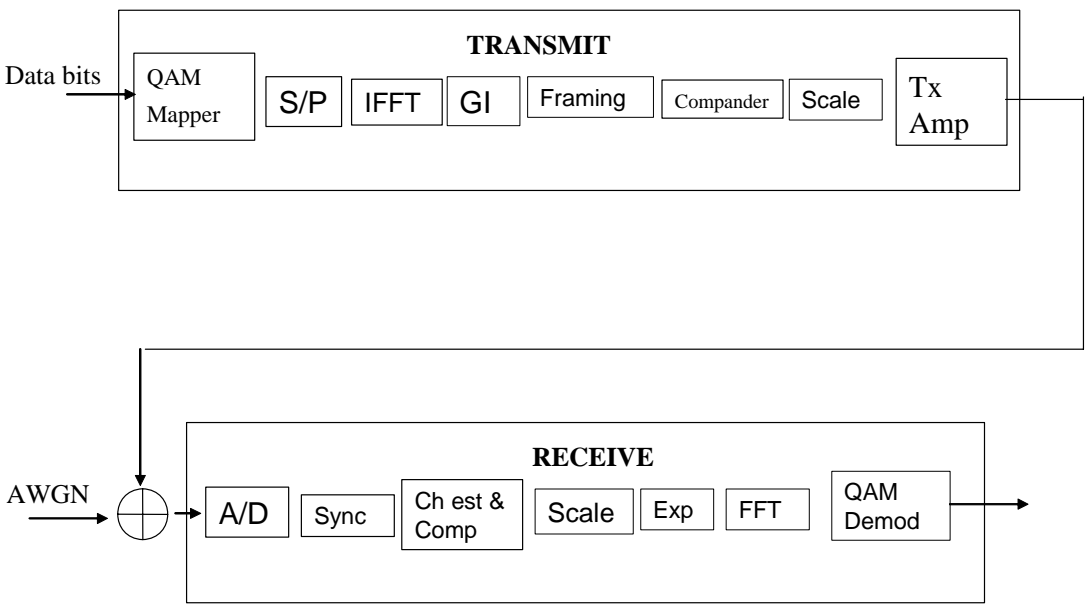

Figure 1. System block diagram 


\section{A. OFDM Signal Model}

The nominal system considered in this study employs $\mathrm{N}_{0}=64$-point OFDM symbols with 52 data carrying subchannels. Let the random complex data QAM symbols be denoted as $S_{\text {dat }}(1), S_{\text {dat }}(2), \ldots, S_{\text {dat }}(52)$. Following [10], we oversample each OFDM block by employing a higher-length DFT with a zero-padded version of the original input signal. Oversampling is employed to address aliasing problems associated with clipping noise, which would fall in-band without oversampling. Using an oversampling rate of $\mathrm{m}=4$ in both the simulations and the hardware testing, the oversampled OFDM signal samples are given by

$$
S_{d a t}(n)=\frac{1}{(52)(3)} \sum_{k=0}^{4 N-1} S_{k} e^{\frac{j 2 \pi n k}{4 N_{\circ}}} \quad 0 \leq n \leq 4 N_{\circ}-1
$$

where

$$
S_{k}=\left\{\begin{array}{cc}
0 & k=0 \\
S_{d a t}(k) & 1<k<26 \\
0 & 27<k<229 \\
S_{d a t}(k-203) & 230<k<255
\end{array}\right.
$$

The scale factor in (1) is included for power normalization.

\section{B. Companding Transform}

Our strategy in this work involves applying $u$-law companding at the transmitter to reduce the PAPR of the transmitted waveform so as to reduce distortion through the transmit amplifier and allow operation closer to amplifier saturation. Values of $u$ ranging between 0.125 and 64 were used in the study since the optimal performance was found to reside within this range of operation.

Let $\mathrm{s}_{\mathrm{dat}}(n)$ be the baseband OFDM signal associated with the data symbol. In the case of $u$-law companding for a selected $u$, the compressed OFDM signal, $\mathrm{s}_{\mathrm{c}}(n)$, is formed as

$$
s_{c}(n)=K(u) s_{\max } \frac{\ln \left[1+u \frac{\left|s_{d a t}(n)\right|}{s_{\max }}\right]}{\ln [1+u]} \operatorname{sign}\left[s_{d a t}(n)\right]
$$

where

$$
S_{\max }=\max _{n}\left(s_{d a t}(n)\right)
$$

and where $\mathrm{K}(u)$ is a normalization constant such that the average power of the companded signal is equal to the average power of the uncompanded signal. A proposed approximation for $\mathrm{K}(u)$ is [5]

$$
K(u) \approx \frac{\ln (1+u)}{u}
$$


However, this approximation is not highly accurate, and in practice, would lead to unnecessary degradation in the demodulation performance. To mitigate errors introduced by normalization inaccuracies, numerically-determined values of K(u) were computed and employed instead, where long-term power averages of both uncompanded and companded OFDM symbols were numerically estimated to find $\mathrm{K}(u)$. The resulting values are plotted in Figure 2 along side the approximation in (5).

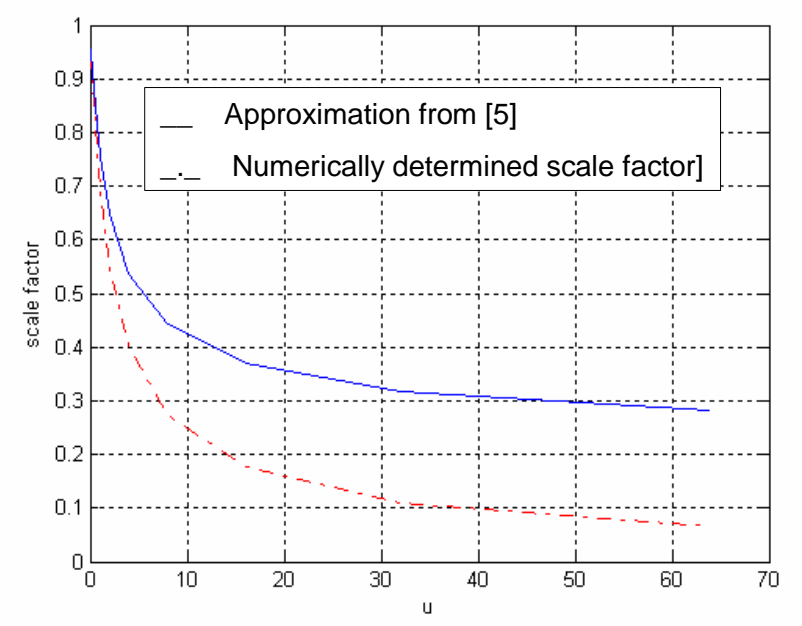

Figure 2. Normalization Constant for Different Values of the Companding
Parameter, with No $=64$ and $4 x$ Oversampling

An example of a time-domain signal associated with a data symbol before and after companding (with $u=16$ ) is shown in Figure 3, where the companded signal is scaled to yield an average power equal to the uncompanded signal. The net result is that companding increases the low-level signal components and reduces the high-level signal components. In the figure, the solid line corresponds to the uncompanded signal, and the dashed line corresponds to the companded signal.

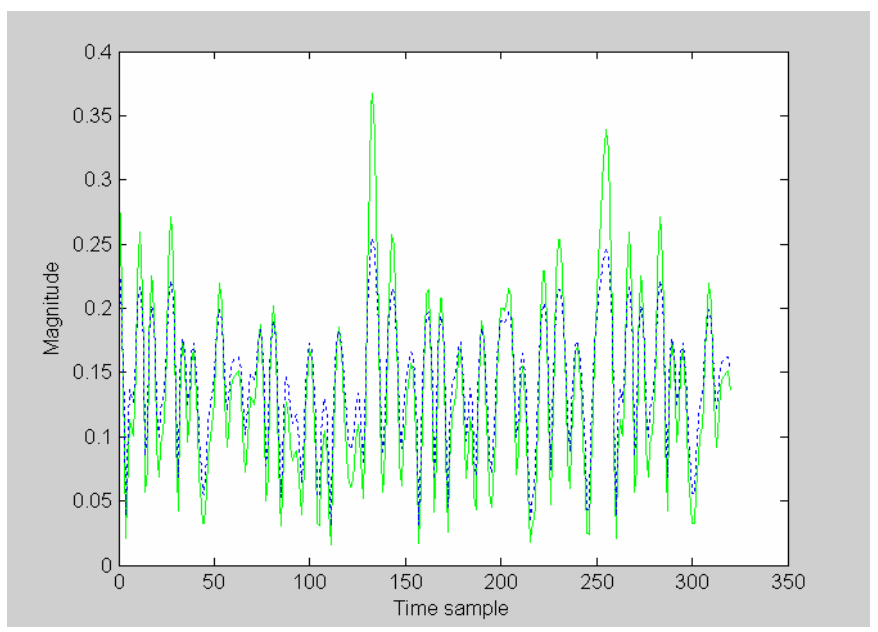

Figure 3. Uncompanded and Companded Signals with Equal Average Power 
Average PAPR associated with the companded signals were also computed as a function of the companding parameters, and are presented in Table 1. For the data symbols, which utilize 52 subcarriers, the use of companding reduces the PAPR by as much as $4 \mathrm{db}$ for the values of $u$ shown in the table.

Table 1. Average PAPR of Data Symbols, with $N_{0}=64$ and $4 x$ Oversampling

\begin{tabular}{|c|c|c|c|}
\hline Companding & PAPR (db) & Companding & PAPR (db) \\
\hline None & 7.3159 & $u=8$ & 5.1055 \\
\hline$u=1$ & 6.7669 & $u=16$ & 4.3361 \\
\hline$u=2$ & 6.3649 & $u=32$ & 3.7014 \\
\hline$u=4$ & 5.8320 & $u=64$ & 3.1175 \\
\hline
\end{tabular}

\section{HPA Nonlinear Models}

The framed packet is then passed through a nonlinear power amplifier model. In this study, a memoryless power amplifier response model [11] is incorporated and the overall link performance is evaluated as a function of the companding parameter, the backoff, and the link SNR. We do this in simulation and then corroborate observed trends in experiments using a software radio testbed.

Representing the complex envelope of the input signal into the amplifier as

$$
s_{c}(t)=\left|s_{c}(t)\right| e^{j \phi(t)}
$$

the transmitted signal at the output of the amplifier may be expressed as [11-13]

$$
S_{t x}(t)=\frac{a\left|s_{c}(t)\right|}{\left[1+\left(\frac{a\left|s_{c}(t)\right|}{A}\right)^{2 p}\right]^{\frac{1}{2 p}}} e^{j \phi(t)}
$$

where $\mathrm{a}$ is the small signal amplification, $\mathrm{A}$ is the saturation output amplitude, and $\mathrm{p}$ is a positive number to control the nonlinear characteristics of the power amplifier model.

In this work, parameter values $\mathrm{p}=2,6$, and 10 are employed. Backoff values are modeled through adjustments in a, and ranged from $0 \mathrm{db}$ (no backoff) to $10 \mathrm{db}$ in the study. To demonstrate the impact that nonlinearities have on symbol errors, received symbol constellations are shown in Figure 4 for infinite SNR with $p=2$ for backoffs of 5 , 10,15, and $20 \mathrm{db}$. Figure 5 shows typical constellations that result for different companding values when the backoff is $5 \mathrm{db}, \mathrm{p}=2$, and the SNR is infinite. The results clearly demonstrate dependence in the error with changes in u. Hence, companding is seen to reduce the distortion from the non-linear amplifier. But use of companding also results in noise amplification when the expansion transform is employed at the receiver. Figure 6 illustrates this effect for the case when SNR $=25 \mathrm{db}$ and a backof of $10 \mathrm{db}$ is employed. The results show that as the companding parameter is increased, the induced symbol noise also increases. 

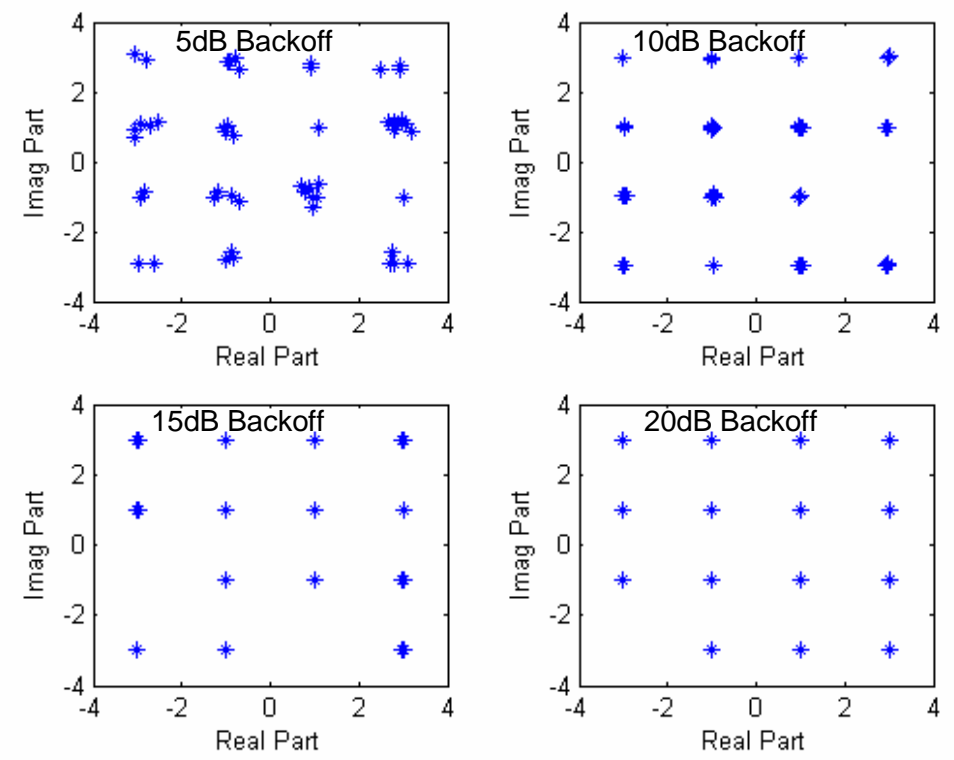

Figure 4. Received Symbol Constellations for a nonlinear SSPA with different backoffs and infinite SNR
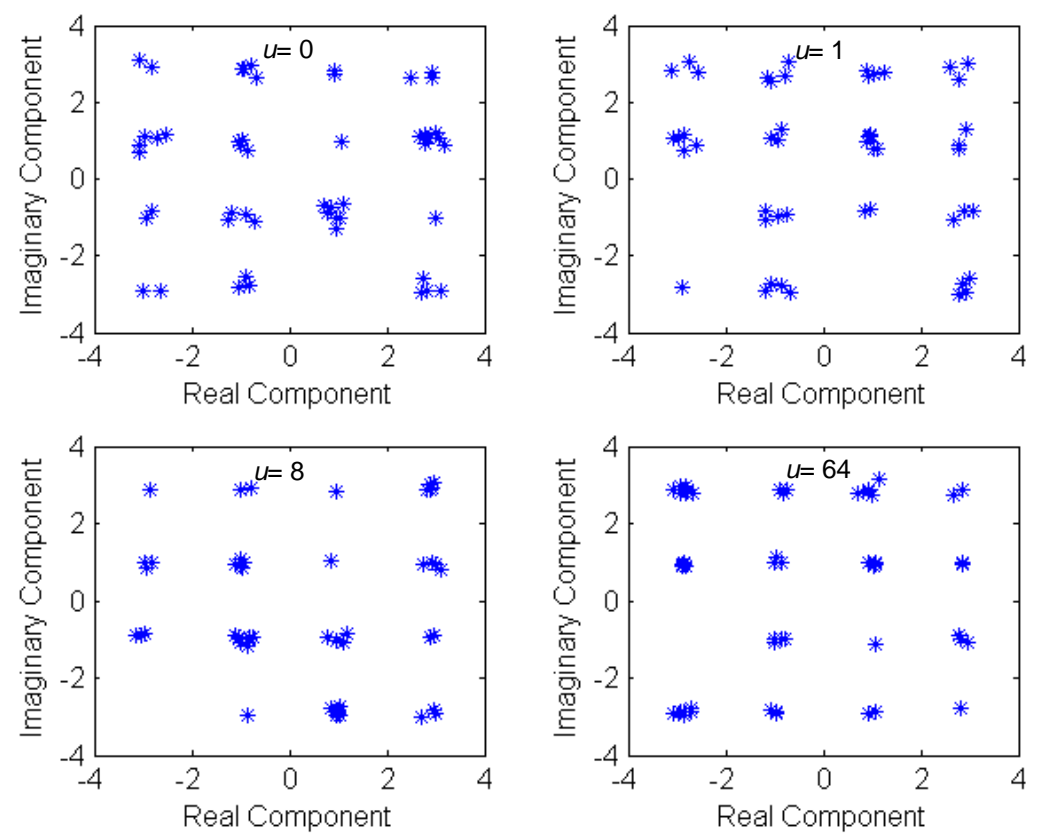

Figure 5. Impact of Increased Companding Parameter on Recovered Symbols, with Infinite SNR, Backoff $=5 \mathrm{db}$, and $\mathrm{p}=10$. 

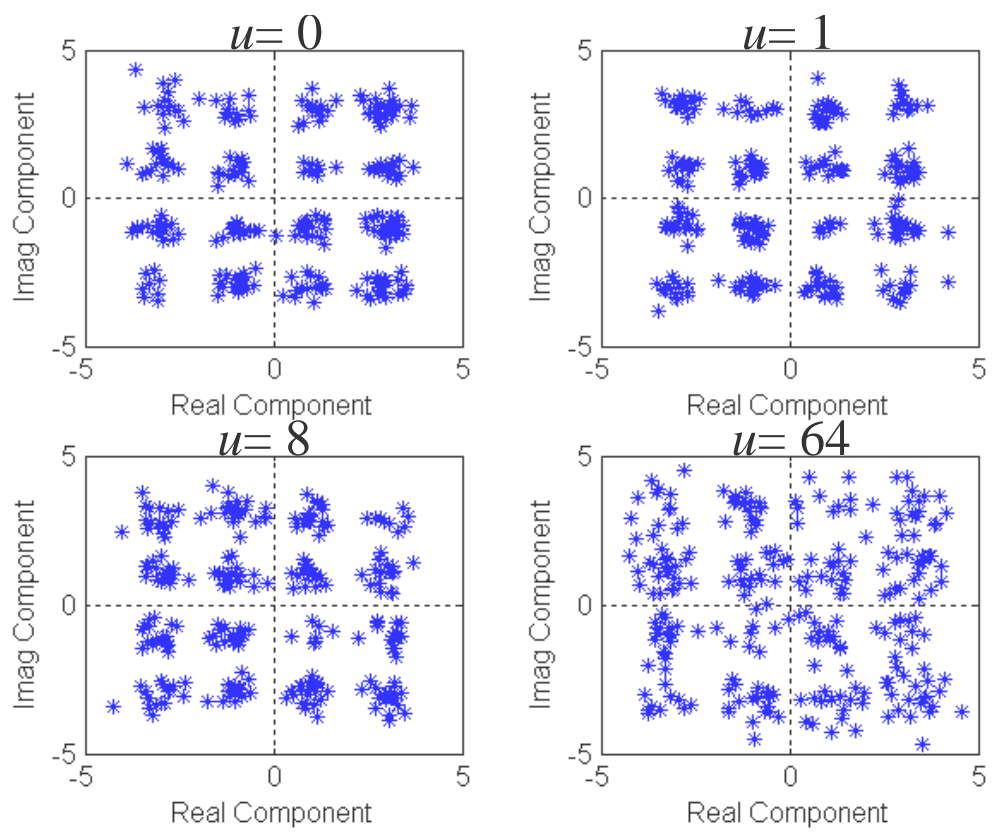

Figure 6. Effect of Noise Amplification on Recovered Symbols, with SNR=25 db, Backoff $=10 \mathrm{db}$, and $\mathrm{p}=10$.

\section{Receiver Processing}

At the receive end, ideal acquisition and tracking are assumed, including ideal sample and symbol synchronization, and perfect frequency estimation. An AWGN channel is assumed, and the noise level is set so that the full-power bandwidth-matched SNR at the receiver (when the transmitter operates at saturation) is either 15, 20, 25, or $30 \mathrm{db}$. The sampled received signal is then represented as

$$
r_{r x}(n)=s_{t x}(n) * h_{c h}(n)+n_{r x}(n)
$$

where $h_{c h}(n)$ is the impulse response of the channel. We assume an all-pass channel, with

$$
h_{c h}(n)=\delta(n)
$$

so that

$$
r_{r x}(n)=s_{t x}(n)+n_{r x}(n)
$$

The received signal is scaled by $\mathrm{K}(\mathrm{u})^{-1}$ and the resulting signal is expanded according to the $u$-law expansion transform. The signal at the output of the expansion transformation is

$$
x(n)=X_{\max }\left(\frac{1}{u}\left((1+u) \frac{K(u)^{-1} r_{r X}(n)}{X_{\max }}-1\right)\right) \operatorname{sign}\left[r_{r x}(n)\right]
$$


The expanded symbol is passed through a serial-to-parallel processor and the resulting blocks are processed through a DFT (with appropriate scaling) to yield

$$
X_{r x}(k)=\sum_{n=0}^{4 N_{o}-1} x(n) e^{-\frac{j 2 m k}{4 N_{o}}} \quad 0 \leq k \leq 4 N_{o}-1
$$

Channel compensation is applied to obtain the recovered QAM symbols $X_{\text {qam }}(\mathrm{k})$ (prior to slicing),

$$
X_{q a m}(k)=X_{r x}(k) H_{c h}(k)=X_{r x}(k)
$$

and these are demodulated to obtain the recovered bit sequence $\mathbf{b}_{\mathrm{rx}}$. Based on these recovered symbols and bits, error metrics are computed for simulation and hardware testing. The error metric used in simulations is the mean symbol error distance

$$
e_{\text {dist }}=E\left\{\mid X_{r x}(k)-S_{t x}(k)\right\} .
$$

This is computed with and without companding for different transmit amplifier backoffs and nominal SNR values to readily identify performance trends. The error metric employed in hardware testing is the bit error rate.

$$
e_{b i t}=E\left\{\left|b_{t x}(n)-b_{r x}(n)\right|\right\}
$$

which is computed for different SNR, backoff values and companding parameter values.

\section{Simulation Results}

The symbol distance error metric with and without companding are presented in Figures 7 through 9 below for SNRs of $25 \mathrm{db}, 30 \mathrm{db}, 35 \mathrm{db}$, and $40 \mathrm{db}$. In each figure, the abscissa represents $\log _{2}(u)$, and the ordinate represents the backoff value in $\mathrm{db}$. Constant error contours are shown, where the contours correspond to the error values associated with each backoff when companding is not used (i.e., when $u=0$ ). For low backoff values, the transmit power is highest, but the performance at the receiver exhibits high error rates due to amplifier nonlinearities. The error rate falls as the backoff decreases but then climbs as the error rates begin to be dominated by low SNR at the receiver.

Each plot clearly shows a region for which operation will provide improved performance over a system without companding. Note that improvements are possible for the range of SNR considered. However, the improvements are generally modest, and reflect an improvement less than roughly $0.5 \mathrm{db}$. 


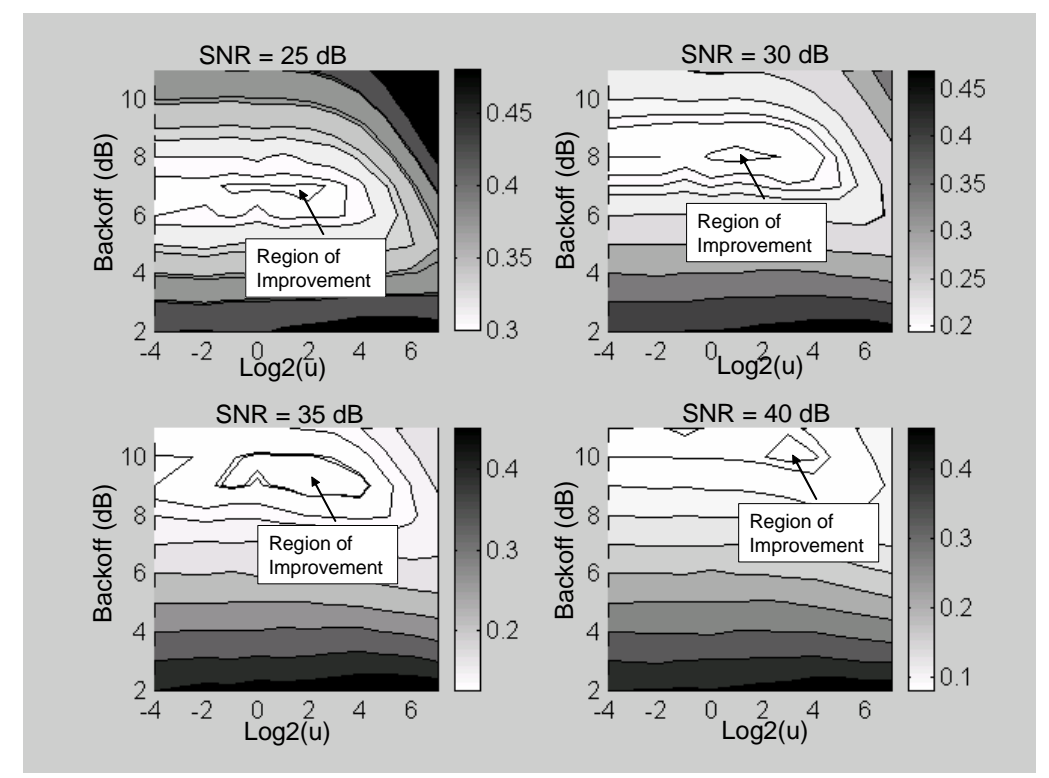

Figure 7. Error metric contours for $\mathrm{p}=2$. SNRs are 25, 30, 35, and $40 \mathrm{db}$

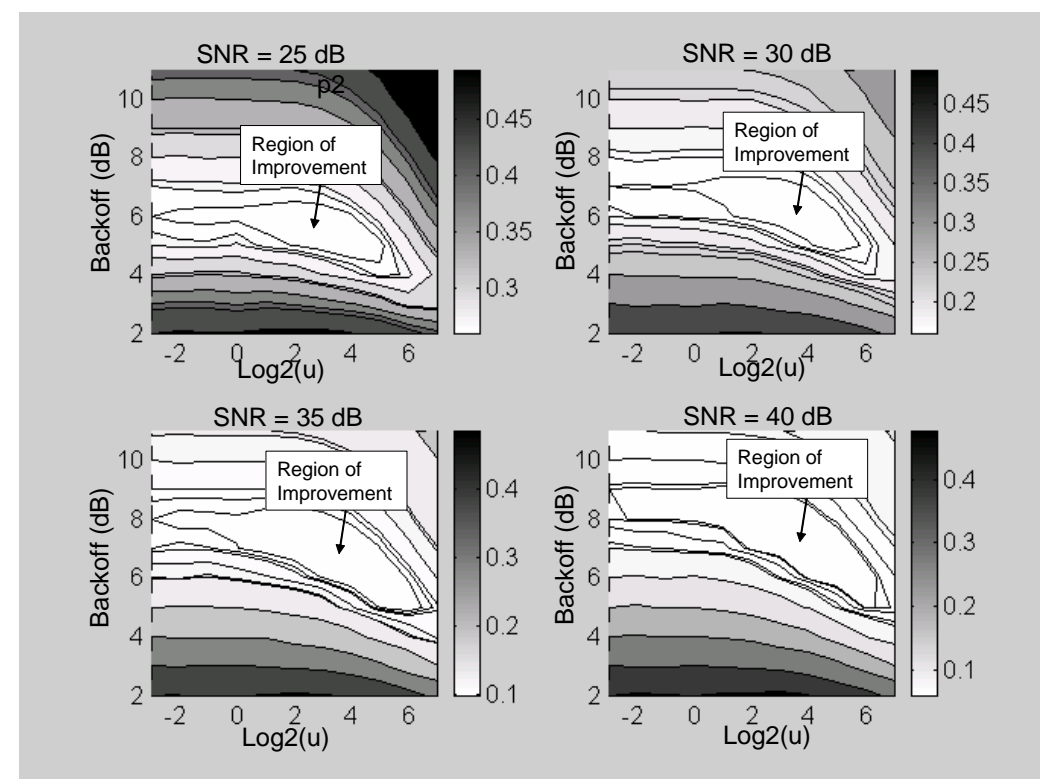

Figure 8. Error metric contours for $\mathrm{p}=6$. SNRs are 25, 30, 35, and $40 \mathrm{db}$ 


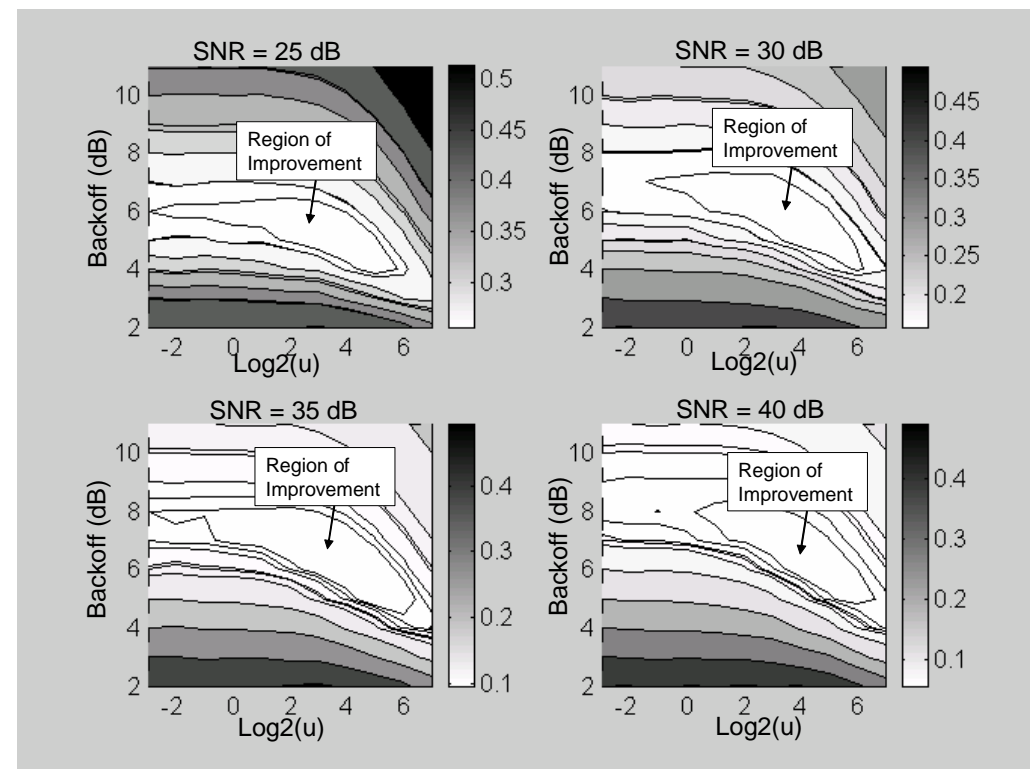

Figure 9. Error metric contours for $\mathrm{p}=10$. SNRs are 25, 30, 35, and $40 \mathrm{db}$

\section{Hardware Testing}

We implemented an OFDM link in a hardware testbed, depicted in Figure 10. Tests included the transmission and reception of over 5 Mbits of data in 25,000 packets, where each packet consisted of a preamble and two training symbols followed by a single OFDM data symbol. The preamble was used for symbol synchronization, and the training symbols for channel estimation. Ideal sampling clock synchronization was achieved in the test bed through use of an external synchronization source provided by an Agilent E4433B ESG-D Series Signal Generator. The transmitted packets each included a single OFDM data symbol to preclude the need for pilot symbols and frequency and/or channel tracking. Significant signal impairments were hence limited to receiver noise, impairments introduced by the transmit amplifier, and to noise amplification introduced by $u$-law expansion at the receiver. The DFT size (256-pt DFT with 64 data subcarriers) in conjunction with zero padding, corresponded to an implementation with 4-times oversampling.

The setup for testing also included a second Agilent E4433B signal generator that was used to generate AWGN. The AWGN waveform was configured with the same channel bandwidth and frequency as the OFDM signal, and the power was set to obtain signal-tonoise ratio of $25 \mathrm{db}$ relative to the transmit signal at saturation. The OFDM signal was generated with Pentek 6229 D/A boards and passed through an amplifier circuit. In order to obtain non-linear characteristics in the input/output power response, a Mini-Circuits ZFL-500 and a ZFL-500HLN were connected in series, where the achieved nonlinearity is due to operation of the second amplifier near saturation. 


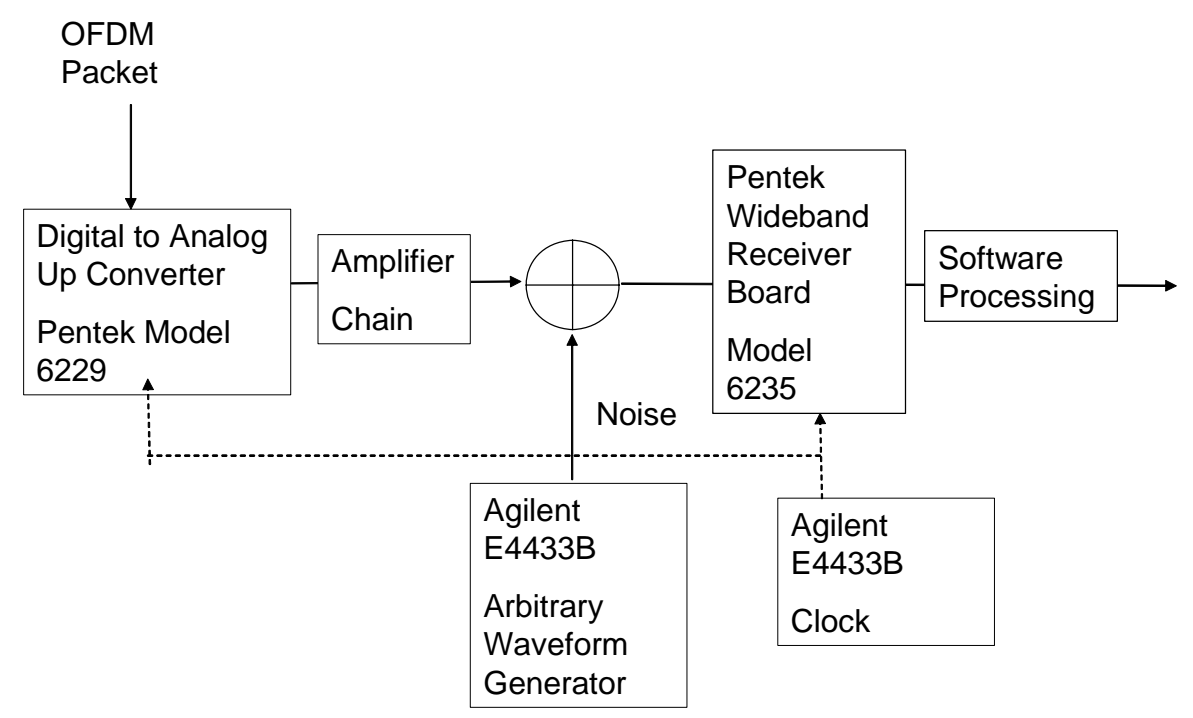

Figure 10. Hardware Test Set-Up

Tests were performed to determine symbol error rates with and without companding and with backoff levels from 0 to 10db. A single test consisted of the transfer of 25,000 OFDM data symbols with 16-QAM symbols loaded on the subcarriers (representing 5.2 Mbits). The data were generated with a pseudorandom sequence generator using the Matlab function 'srand' with a controlled seed so that the data could be reproduced for comparison purposes later. After the data symbols were received and demodulated, the resulting data values were passed via an FPDP I/O link to a PC in order to compute the bit error rates for each test. Since the data were produced with a seeded pseudorandom number generator, the values were easily regenerated for comparison to compute the bit error rate for each test run. The testbed implemented the $u$-law transforms and tests were performed using values for $u$ that included $0,0.125,0.25,0.5,1,2,4$, and 8 .

Table 2. Bit Error Count for 25,000 OFDM symbols with SNR $=25 \mathrm{db}$ SNR 25

\begin{tabular}{|c|c|c|c|c|c|c|}
\hline Mu & $0 \mathrm{db}$ & $-1 d b$ & $-2 d b$ & $-5 d b$ & $-7 \mathrm{db}$ & $-10 d b$ \\
\hline 0 & 59559 & 37302 & 21318 & 2825 & 1306 & 5055 \\
\hline 0.125 & 61230 & 37681 & 21791 & 2856 & 1236 & 5095 \\
\hline 0.25 & 62374 & 39554 & 22655 & 3036 & 1277 & 5124 \\
\hline 0.5 & 64899 & 40561 & 23520 & 3110 & 1379 & No Sync \\
\hline 1 & 68469 & 43567 & 25622 & 3368 & 1489 & No Sync \\
\hline 2 & 76082 & 49012 & 28631 & 3816 & 1724 & No Sync \\
\hline 4 & 89387 & 57169 & 34393 & 4711 & 2007 & No Sync \\
\hline 8 & 106478 & 70666 & 42337 & 6153 & 2692 & No Sync \\
\hline
\end{tabular}

The results from the hardware testing, shown in Table 2, reflect similar trends that were observed in the simulation study. Namely, companding provides modest gains in 
performance at backoff levels slightly less than or equal to the backoff providing best performance when companding is not used. For the particular amplifier set-up used in the tests, performance gains are achieved at the same backoff that optimizes performance without companding, and companding values of 0.125 and 0.5 yielded gains over the non-companded case. These realized performance gains come at a price of increased computational load at both the transmitter and the receiver.

\section{Conclusions}

We have investigated the link performance of OFDM with companding as a PAPR mitigation strategy. Impairments from nonlinear distortion at the transmitter, AWGN noise from the channel, and noise amplification due to the expansion transform at the receiver were considered. Simulation and hardware testing were employed to investigate performance trends as a function of companding parameters, amplifier nonlinearities, backoff, and SNR. The simulation tests utilized a symbol error distance metric, while hardware testing utilized bit error metrics. The results from the study show that at low backoffs, impairments from nonlinear amplification can be significant. Increasing the compression (via the companding parameter, $u$ ) reduces the non-linear distortion and suggests that moving the operating point towards saturation might be beneficial. However, increasing the companding parameter also leads to noise amplification during expansion at the receiver, which potentially can largely negate any performance gains that would otherwise accrue from higher values of $u$. Thus, the companding parameter and backoff should be chosen to balance these competing effects. In simulation analyses it was found that a system incorporating companding can yield improved performance over a system that does not employ companding. However, the performance gains are modest (around $0.5 \mathrm{db}$ ) and add processing complexity at both the transmit and receive ends of the link.

These results are also consistent with the performance trends presented in [20,21]. Namely, for high values of $u$, the performance of the system without companding outperforms the system with companding. However, as we have seen, with an appropriate choice of $u$ and amplifier backoff, the companding system can outperform a system without companding. The same may hold for other companding approaches, such as those proposed in [20,21].

\section{Acknowledgements}

Any opinions, finding, and conclusions or recommendations expressed in this material are those of the authors and do not necessarily reflect the views of The National Science Foundation.

\section{References}

1. R.Van Nee and R. Prasad, OFDM for Wireless Multimedia Communications, Artech House Publishers, 2000.

2. P. Struhsaker and K. Griffin, "Analysis of PHY waveform Peak to Average Power Ratio and Impact on RF Amplification,” IEEE802.163c-01/46, March 9, 2001.

3. A.G. Armada and J.L. Garcia, "Admissible spectral re-growth in OFDM-based broadband WLAN.

4. X. Wang, T. Tjhung, and C. Ng, "Reduction of peak-to-average power ratio of OFDM system using a companding technique, IEEEE Transactions of Broadcasting, Vol, 45m, No.3, Sept. 1999. 
5. A. Mattsson, G. Mendenhall, and T. Dittmer, "Comments on Reduction of peak-to-average power ratio of OFDM system using a companding technique,” IEEE Transaction on Broadcasting, vol. 45. No. 4. December 1999.

6. X. Wang, T Tjhung, and C. Ng, "Reply to the Comments on Reduction of peak-to-a Average power ratio of OFDM system using a companding technique," IEEE Transactions on Broadcasting, Vol. 45, No. 4 December 1999, p. 420-423.

7. X. Wang, T. Tjhung, C. Ng, and A. Kassim, "On the SER Analysis of A-Law Companded OFDM System, Global Telecommunications Conference, 2000. GLOBECOM '00. IEEE, Vol. 2, Dec. 2000, p. $756-760$.

8. X. Huang, J. Lu, J. Chuang, and J Zheng, "Companding Transform for the Reduction of Peak-toAverage Power Ratio of OFDM Signals,” IEEE VTC 20001, p. 835-839.

9. X. Huang, J. Lu, J. Zheng, J. Chuang and J. Gu, "Reduction of peak-to-average power ratio of OFDM signals with companding transform,” Electronics Letters, 12 April 2001, vol. 37, No.8, p 506-507.

10. X. Li and L Cimini Jr., "Effects of Clipping and Filtering on the Performance of OFDM," IEEE 1997, p. 1634-1638.

11. Rapp, C. "Effects of HPA Nonlinearity on a 4-DPSK/OFDM Signal for a Digital Sound Broadcasting System," Proceedings of the Second European Conference on Satellite Communications, Liege, Belgium, pp. 179-184, Oct 22-24, 1991.

12. N. Guo, and L. Milstein,”The impact of Nonlinear Amplification on Multi-Code CDMA Systems,” IEEE 2000, p. 1034-1038.

13. G. Santella and F. Mazzenga, "A hybrid analytical simulation procedure for performance evaluation in M-QAM OFDM schemes in presence of nonlinear distortions,” IEEE Trans. Vehicular Tech,., vol. 47, pp. 142-151, Feb 1998.

14. J. Davis and J. Jedwab, "Peak-to-Mean Power Control in OFDM, Golay Complementary Sequences, and Reed-Muller Codes," IEEE Transactions on Information Theory, Vol. 45, No. 7, November 1999, p. 2397-2417.

15. H.H. Seung and H.L. Jae, "Modified selected mapping technique for PAPR reduction of coded OFDM signal,” IEEE Trans. Broadcast., vol. 50, no. 3, pp.335-341, Sep. 2004

16. S.H. Wong, A. S. Madhukumar, and F. Chin, "Peak-to-average power reduction using partial transmit sequences: a suboptimal approach based on dual layered phase sequencing," IEEE Trans. Broadcast., vol. 49, no. 2, pp. 225-231, Jun. 2003.

17. B.S. Krongold an $\mathrm{dD}$. L. Jones, "PAPR reduction in OFDM via active constellation extension," IEEE Trans. Broadcast., vol. 49, no. 3, pp. 258-268, Sept 2003.

18. Z. Yang, H. Fang, and C. Pan, "ACE with frame interleaving scheme to reduce peak-to-average power ratio in OFDM systems,” IEEE Trans. Broadcast., vol. 51, no. 4, pp. 571-575, Dec. 2005.

19. D. W. Lim, S. J. Heo, J. S. No, and H. Chung, "A New PTS OFDM Scheme with Low Complexity for PAPR Reduction,” IEEE Trans. Broadcast, Accepted for future publication.

20. T. Jiang, Y. Yang, and Y. Song, "Exponential companding transform for PAPR reduction in OFDM systems, IEEE Trans. Broadcast., vol. 51, no. 2, pp. 244-248, Jun. 2005.

21. T. Jiang and G. Zhu, "Nonlinear companding transform for reducing peak-to-average power ratio of OFDM signals, "IEEE Trans. Broadcast., vol. 50, no. 3, pp. 342-346, Sep. 2004. 